\title{
Analytical Model and Numerical Simulation for the Effect of Uncertain Initial Geometrical Imperfection on the Buckling of Thin Plate
}

\author{
Shu Zhang ${ }^{1, a)}$, Hao Xiao ${ }^{2, b)}$, Fangwei Qiang ${ }^{1, \mathrm{c})}$, Weiping $\mathrm{Hu}^{3, \mathrm{~d})}$ \\ ${ }^{1}$ Beijing Aerospace Times Laser Inertial Technology Company, LTD, Beijing 100094, China \\ ${ }^{2}$ Beijing Aeronautical Science \& Technology Research Institute, Beijing 102211, China \\ ${ }^{3}$ School of Aeronautics Science and Engineering, BeiHang University, Beijing 100191, China \\ a)buaazs17@163.com, ${ }^{\text {b) }}$ hotboyhao@gmail.com, ${ }^{\mathrm{c})}$ qiangfangwei@163.com, ${ }^{\mathrm{d})}$ huweiping@buaa.edu.c
}

$\mathrm{n}$

Keywords: buckling; initial geometrical imperfection; double trigonometric series; uncertainty; Monte Carlo simulation

\begin{abstract}
Due to the uncertainty of the initial geometrical imperfection, it is difficult to evaluate its influence on bucking behavior of structures. In this study, the double trigonometric series is adopted to describe the initial geometric imperfection and the deformed deflection, and then the relation between them is deduced from the bending equilibrium equation. Therefore, the total displacement can be obtained by superposing these two deflections. Based on this analytical model, the range of the maximum deformation is determined by Monte Carlo simulation. The analysis provides a new analytical model for the buckling problem of plate with uncertain initial geometrical imperfection.
\end{abstract}

\section{INTRODUCTION}

Previously reported experimental studies [1] show that the experimental values of critical load are much lower and the data has a larger scatter band when compared with the results predicted by the linear theories of buckling. It is widely accepted that the result is caused by the original defects of structures. These original defects mainly include the thickness imperfection [2, 3], misalignment in the loading [4], non-perfect boundary conditions [5], fluctuations in material properties [6], impact of residual stress [7] and the initial geometrical imperfection [8-13], and among which the initial geometrical imperfection is the major one whose influence on buckling has become a very important issue [14].

Flügge [15] firstly studied the effect of initial geometrical imperfection on the shell stability based on linear buckling equations, however, he could not make reasonable explanations because that equation can not describe the deformation of post-buckling. Donnell studied the post-buckling equilibrium path of cylindrical shell by assuming the initial geometrical imperfection as a slight deviation from the ideal cylindrical shell and is of the same shape with the buckling deflection [16], and described the impact of the initial geometrical imperfection on buckling load by proposing an initial defect factor. Later Koiter [17] put forward a post-buckling theory to depict the initial post-buckling behavior with imperfection sensitivity and introduced a precise theory which results in an asymptotically solution. Hutchinson presented a two equivalent description of initial post-buckling theory based on the energy principle and the virtual work principle $[18,19]$, which extended the application scope of Koiter theory.

However the initial geometrical imperfection is of random shape and random amplitude in engineering application, the aforementioned theory can not take the uncertainty of initial geometrical imperfection into account. There are two methods to solve this problem, one is that calculate the buckling load through harmonic analysis based on the deflection distribution measured by actual measurement, and the other is that establish an appropriate criterion for buckling by analyzing the effect of the random initial geometrical imperfection on buckling behavior via stochastic theory. Since the former method needs plenty of measurement data, more research focuses on the latter. The stochastic theory includes non-probability convex model method and probability method. Convex model method is adopted to analyze the problem of structure with 
uncertain initial geometrical imperfection in Literatures [20, 21], and the interval estimation of safety factor is given. However this method still needs some experiment data, which is difficult to be measured, to determine the parameters depicting the imperfection. Probability method establishes the model by taking the uncertainty as a random variable. In Elishakoff's study [22], the initial geometrical imperfection on the rod is expressed by Fourier series, which has the same form of the buckling mode, and the coefficients are randomly given to depict the random defect. On the basis of that model, some numerical methods, for example, Monte Carlo method [23], can be used to calculate the buckling behavior of the structure.

In this study, double trigonometric series, whose coefficients are randomly selected within the specific range that are defined according to the manufacturing process error, is adopted to describe the initial geometric imperfection of plate with four edges simply supported. Then the deformed deflection can be solved from the bending equilibrium equation of thin plate and the maximum deflection is calculated to evaluate the stability of structure based on Hoff safety criterion. The Monte Carlo method and finite element method are applied to the numerical simulation for random response of the buckling behavior.

\section{BASIC EQUATIONS AND METHOD OF SOLUTION}

A square plate with four edges simply supported is studied in this section. The length of the edge $a$ is equal to $40 \mathrm{~mm}$, and the thickness of the plate $\mathrm{h}$ is equal to $0.6 \mathrm{~mm}$. The material of plate is LC9CgS1 aluminum alloy whose static properties are listed in Table 1.

TABLE 1. Static properties of LC9CgS1 aluminum alloy material

\begin{tabular}{ccc}
\hline Parameter & $\mathbf{E}(\mathbf{G P a})$ & $\mu$ \\
\hline Value & 70 & 0.3 \\
\hline
\end{tabular}

Two opposite edges of plate are loaded by uniform pressure $P_{x}$ equaling to $40 \mathrm{~N} / \mathrm{mm}$. The geometrical shape and loading conditions are shown in Figure.1.

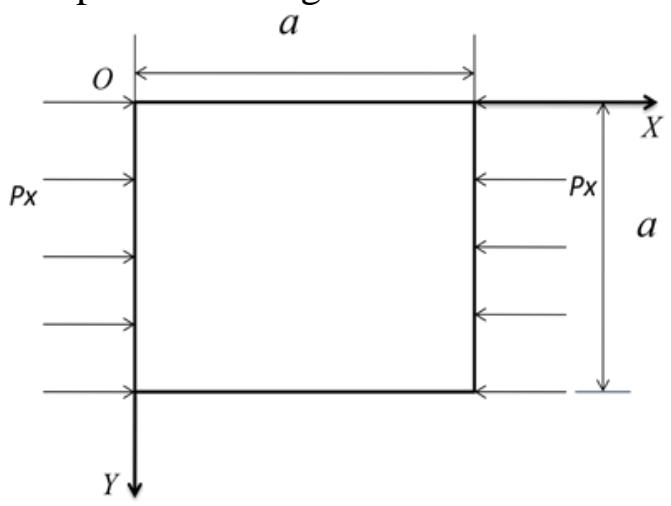

FIGURE 1. The geometrical shape and loading of plate

Boundary conditions are expressed as follows:

$$
\begin{cases}x=0, a & w=0, \frac{\partial^{2} w}{\partial x^{2}}=0 \\ y=0, a & w=0, \frac{\partial^{2} w}{\partial y^{2}}=0\end{cases}
$$

The deflection equilibrium equation of simply supported plate under unidirectional pressure is expressed as follows:

$$
D\left(\frac{\partial^{4} w}{\partial x^{4}}+2 \frac{\partial^{4} w}{\partial x^{2} \partial y^{2}}+\frac{\partial^{4} w}{\partial y^{4}}\right)+P_{x} \frac{\partial^{2} w}{\partial x^{2}}=0
$$

where $D=E h^{3} /\left[12\left(1-\mu^{2}\right)\right]$ is the flexural rigidity of the plate.

The deflection function can be expressed by double trigonometric series as follows: 


$$
w=\sum_{m=1}^{\infty} \sum_{n=1}^{\infty} A_{m n} \sin \frac{m \pi x}{a} \sin \frac{n \pi y}{a}(m, n=1,2,3, \cdots)
$$

Substituting equation (3) into (2), then we have:

$$
\sum_{m=1}^{\infty} \sum_{n=1}^{\infty} A_{m n}\left[\frac{m^{4} \pi^{4}}{a^{4}}+2 \frac{m^{2} n^{2} \pi^{4}}{a^{4}}+\frac{n^{4} \pi^{4}}{a^{4}}-\frac{P_{x}}{D} \frac{m^{2} \pi^{2}}{a^{2}}\right] \sin \frac{m \pi x}{a} \sin \frac{n \pi y}{a}=0
$$

The above equation indicates that:

$$
A_{m n}\left[\pi^{4}\left(\frac{m^{2}}{a^{2}}+\frac{n^{2}}{a^{2}}\right)^{2}-\frac{P_{x}}{D} \frac{m^{2} \pi^{2}}{a^{2}}\right]=0
$$

Because $A_{m n}$ equaling to 0 means the plate maintains the state of plane equilibrium under all kinds of load conditions, which is impossible, then the letter term next to $A_{m n}$ must be equal to 0 . That is:

$$
\pi^{4}\left(\frac{m^{2}}{a^{2}}+\frac{n^{2}}{a^{2}}\right)^{2}-\frac{P_{x}}{D} \frac{m^{2} \pi^{2}}{a^{2}}=0
$$

Critical buckling load of perfect plate is therefore derived as follows:

$$
\left(P_{x}\right)_{c r}=\frac{D a^{2} \pi^{2}}{m^{2}}\left[\frac{m^{2}}{a^{2}}+\frac{n^{2}}{a^{2}}\right]^{2}
$$

The first order buckling load is $34.2 \mathrm{~N} / \mathrm{mm}$ in this case, which means structure is in post-buckling state since the external load $P_{x}$ is $40 \mathrm{~N} / \mathrm{mm}$.

In this study the random initial geometrical imperfection is also expressed by double trigonometric series as follows

$$
w_{0}=\sum_{m=1}^{\infty} \sum_{n=1}^{\infty} B_{m n} \sin \frac{m \pi x}{a} \sin \frac{n \pi y}{a}(m, n=1,2,3, \cdots)
$$

By introducing dimensionless quantities as follows

$$
\overline{w_{0}}=\frac{w_{0}}{a}, \bar{w}=\frac{w}{a}, \xi=\frac{x}{a}, \eta=\frac{y}{a}
$$

The deflection equilibrium equation of simply supported plate with initial geometrical imperfection under unidirectional pressure can be obtained as follows

$$
D\left(\frac{\partial^{4} \bar{w}}{\partial \xi^{4}}+2 \frac{\partial^{4} \bar{w}}{\partial \xi^{2} \partial \eta^{2}}+\frac{\partial^{4} \bar{w}}{\partial \eta^{4}}\right)+P_{x} a^{2} \frac{\partial^{2}\left(\bar{w}+\overline{w_{0}}\right)}{\partial \xi^{2}}=0
$$

We introduce the function of $G(m, n)$ and assume that

$$
A_{m n}=B_{m n} G(m, n)
$$

Substituting equations (3), (8), (9) and (11) into equation (10), we can get the following as

$$
\sum_{m=1}^{\infty} \sum_{n=1}^{\infty} A_{m n}\left\{\pi^{4}\left(m^{2}+n^{2}\right)^{2}-\frac{1}{G} \frac{P_{x} a^{2}}{D} m^{2} \pi^{2}-\frac{P_{x}}{D} m^{2} \pi^{2} a^{2}\right\} \sin m \pi \xi \sin n \pi \eta=0
$$

It is clear from equation (12) that

By solving equation (13) yields

$$
\pi^{4}\left(m^{2}+n^{2}\right)^{2}-\frac{1}{G} \frac{P_{x} a^{2}}{D} m^{2} \pi^{2}-\frac{P_{x}}{D} m^{2} \pi^{2} a^{2}=0
$$

$$
G(m, n)=\frac{P_{x} a^{2} m^{2}}{\pi^{2}\left(m^{2}+n^{2}\right)^{2} D-P_{x} a^{2} m^{2}}
$$

Thus the total non-dimensional out-of-plate deflection is given as follows

$$
\overline{w_{f}}=\bar{w}+\overline{w_{0}}=\sum_{m=1}^{\infty} \sum_{n=1}^{\infty} B_{m n}(1+G(m, n)) \sin m \pi \xi \sin n \pi \eta(m, n=1,2,3, \cdots)
$$

Therefore, the maximum out-of-plate deflection can be obtained as follows 


$$
\max w_{f}=\max \sum_{m=1}^{\infty} \sum_{n=1}^{\infty} a \cdot B_{m n}(1+G(m, n)) \sin m \pi \xi \sin n \pi \eta(m, n=1,2,3, \cdots)
$$

If we confine the amplitude of initial geometrical imperfection into the range of $(-\varepsilon, \varepsilon)$ according to the manufacture process error, and introduce that

$$
\bar{\varepsilon}=\frac{\varepsilon}{a}
$$

Then the constraint function can be expressed as follows:

$$
-\bar{\varepsilon}<\overline{w_{0}}=\sum_{m=1}^{\infty} \sum_{n=1}^{\infty} B_{m n} \sin m \pi \xi \sin n \pi \eta<\bar{\varepsilon}
$$

A group of $B_{m n}$ is randomly selected according to the constraint function (18), then the maximum deflection is calculated by equation (16). The probability distribution of safety factor is further determined based on the distribution of maximum deflection and the allowable value of deflection.

According to Hoff safety criterion [24], if the maximum out-of-plate displacement of structure exceeds the allowed value $Q$, then the structure loses effectiveness. The allowed value $Q$ is a critical point of failure. The buckling safety factor is defined as follows

$$
f(\xi, \eta)=\frac{Q}{\max w_{f}}
$$

where $\max { }^{w_{f}}$ represents maximum out-of-plate displacement, $|f(\xi, \eta)|>1$ means structure is safe, and $|f(\xi, \eta)|<1$ means structure is unstable.

\section{MONTE CARLO SIMULATION}

On the basis of the analytical model presented in section 2, a group of $B_{m n}$ randomly selected according to the restriction condition of equation (18) corresponds to a kind of initial geometrical imperfection. The value of max $\overline{w_{f}}$ corresponding to each group of $B_{m n}$ is then calculated and the range of $f(\xi, \eta)$ can be obtained by stochastic simulations.

For the case in this paper, set $\varepsilon=0.0001 \mathrm{~mm}$ and $\bar{\varepsilon}=2.5 \times 10^{-6}$.

$G(m, n)$ can be calculated as follows

$$
G(m, n)=\frac{m^{2}}{0.218\left(m^{2}+n^{2}\right)^{2}-m^{2}}
$$

Then the total non-dimensional out-of-plate deflection is expressed as follows

$$
\overline{w_{f}}=\sum_{m=1}^{\infty} \sum_{n=1}^{\infty} B_{m n}\left(\frac{0.218\left(m^{2}+n^{2}\right)^{2}}{0.218\left(m^{2}+n^{2}\right)^{2}-m^{2}}\right) \sin m \pi \xi \sin n \pi \eta(0 \leq \xi, \eta \leq 1)
$$

The results show that when the number of terms of double trigonometric series reaches 100, i.e. $m_{\max }=10, n_{\max }=10$, the deflection of plate is convergent. So all the results in this paper are obtained in the condition of $m_{\max }=10, n_{\max }=10$.

A typical initial geometrical imperfection is shown in Figure.2. And the interval of maximum deflection which is convergent after simulating 1000 times is shown in Figure.3 and Figure.4. 


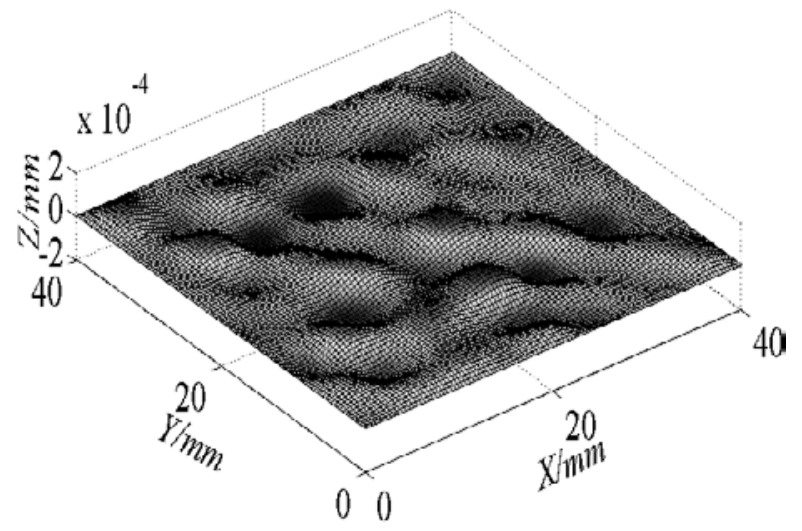

FIGURE 2. A typical initial geometrical imperfection

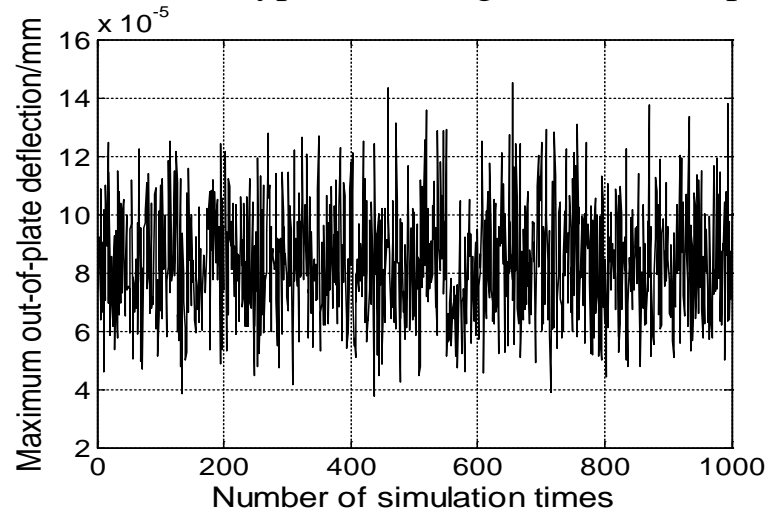

FIGURE 3. Maximum deflection in the normal direction by Monte Carlo simulation

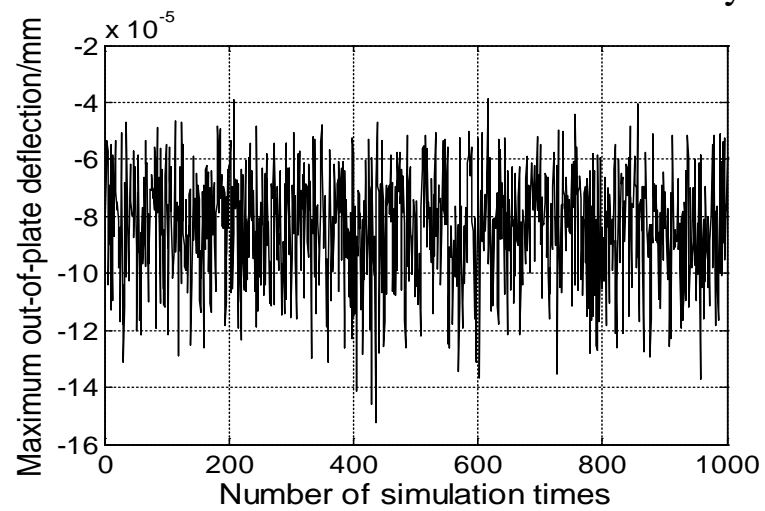

FIGURE 4. Maximum deflection in the opposite normal direction by Monte Carlo simulation The result shows the maximum deflection in the normal direction is $\left(3.85 \times 10^{-5} \mathrm{~mm}, 1.51 \times 10^{-4}\right.$ $\mathrm{mm})$, and that in the opposite direction is $\left(-1.51 \times 10^{-4} \mathrm{~mm},-3.85 \times 10^{-5} \mathrm{~mm}\right)$. So the range of maximum deflection is $\left(-1.51 \times 10^{-4} \mathrm{~mm}, 1.51 \times 10^{-4} \mathrm{~mm}\right)$.

$Q$ is set to be $1 \times 10^{-4} \mathrm{~mm}$ in this paper, and then the zone between the two horizontal dotted lines is defined as the failure zone, which is illustrated in Figure.5. The distribution of safety factor is illustrated in Figure.5 and Figure.6. 


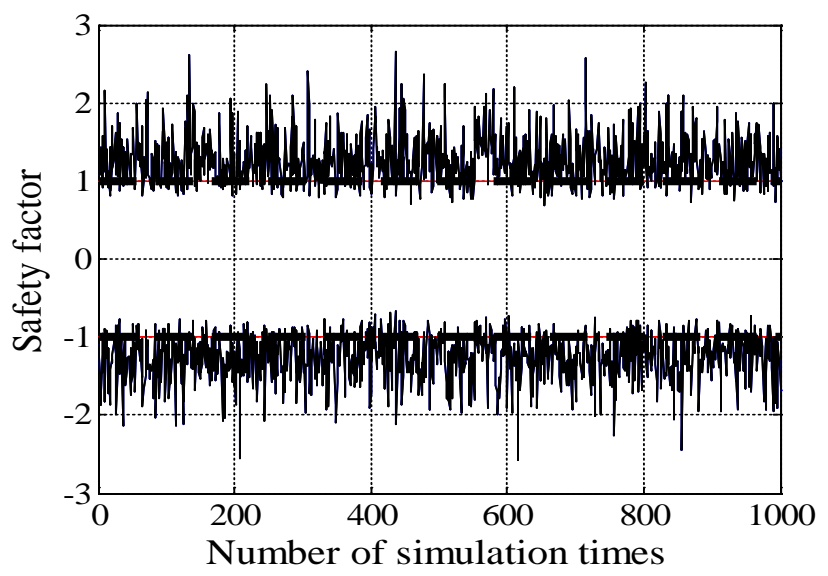

FIGURE 5. Safety factor curve

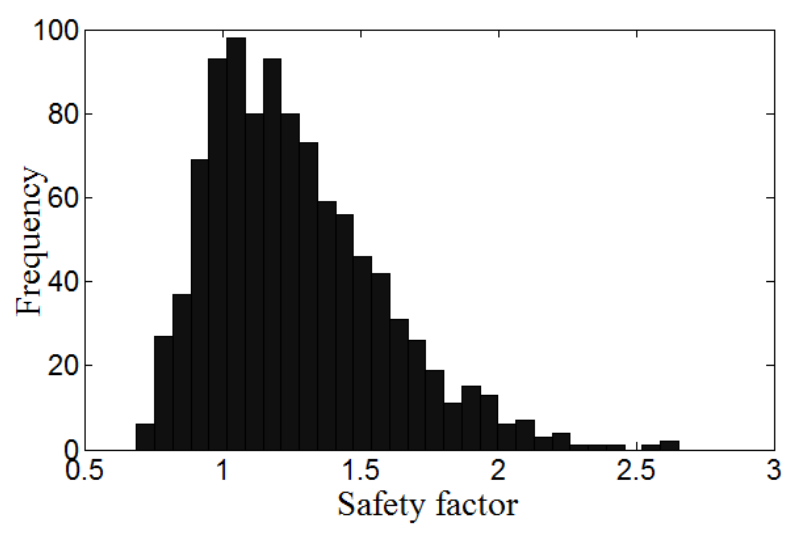

FIGURE 6. Histogram of safety factor in the normal direction of plate

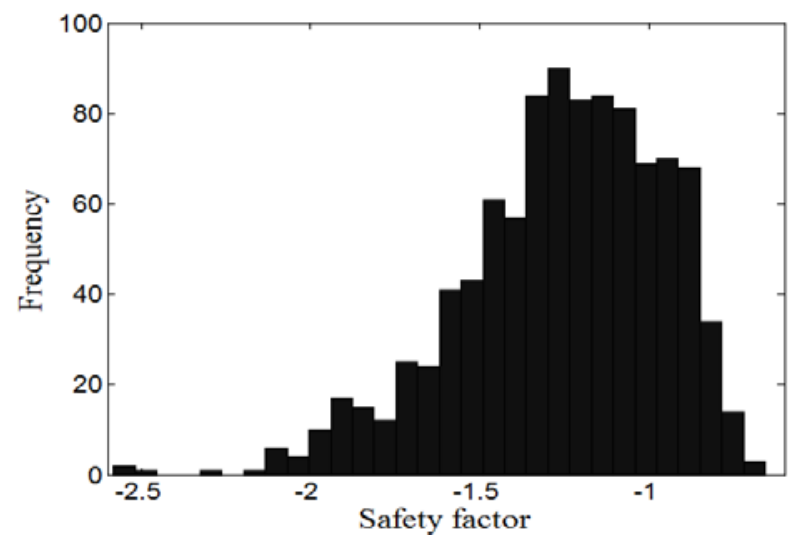

FIGURE 7. Histogram of safety factor in the opposite normal direction of plate

According to the calculating results, the probability distribution of safety factor is obtained, which are shown in Figure.7 and Figure.8. There are 213 times of total 1000 times simulation for $|f(\xi, \eta)|<1$, so the failure probability of structure is equal to $21.3 \%$.

\section{FINITE ELEMENT SIMULATION}

The initial geometrical imperfection applied on structure to be simulated by finite element method must be certain, then the first 200 buckling modes calculated by eigenvalue buckling analysis are applied to the original structure as the shape of initial defect with the amplitude taken as $0.0001 \mathrm{~mm}$. The maximum deflection for each simulation is recorded and displayed in Figure.8 and Figure.9. 


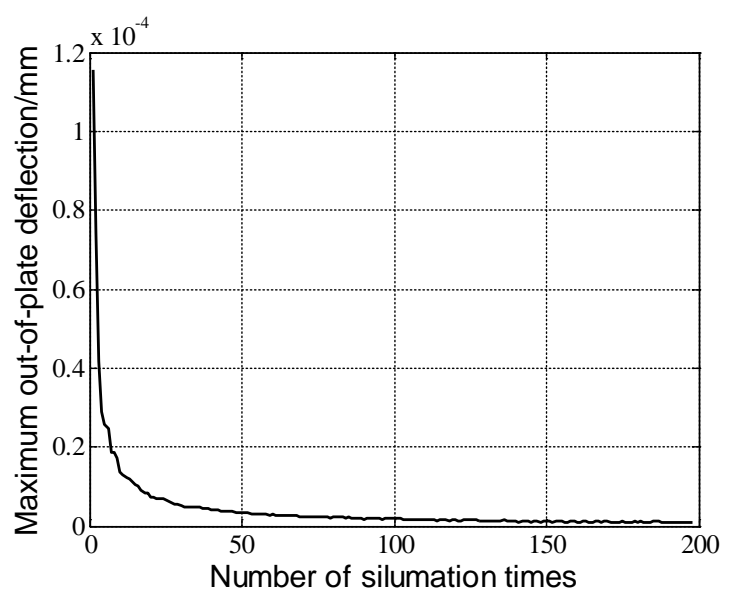

FIGURE 8. Maximum deflection in the normal direction by finite element calculation

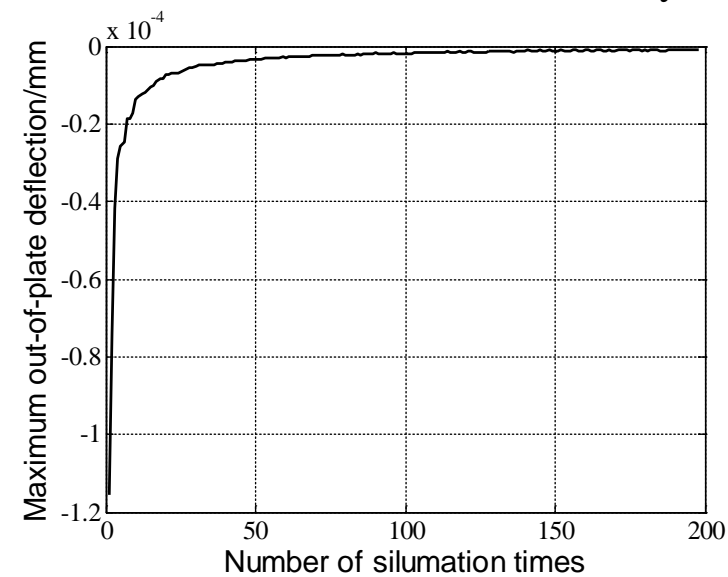

FIGURE 9. Maximum deflection in the opposite normal direction by finite element calculation The range of maximum deflection in the normal direction is $\left(0,1.15 \times 10^{-4} \mathrm{~mm}\right)$, while that in the opposite direction is $\left(-1.15 \times 10^{-4} \mathrm{~mm}, 0\right)$. So the interval of maximum deflection is $\left(-1.15 \times 10^{-4}\right.$ $\left.\mathrm{mm}, 1.15 \times 10^{-4} \mathrm{~mm}\right)$ which is in the range of $\left(-1.51 \times 10^{-4} \mathrm{~mm}, 1.51 \times 10^{-4} \mathrm{~mm}\right)$ given by Monte Carlo simulation. This result is reasonable since the geometrical imperfection applied in finite element method is the part of all probable imperfections.

\section{CONCLUSIONS}

A new method is proposed to depict the initial geometrical imperfection and to evaluate the failure probability of the structure. The initial geometrical imperfection which has the same form of bending deflection of plate is expressed by double trigonometric series, then the relationship between the coefficients of bending deflection and initial geometrical imperfection can be derived by solving the deflection equilibrium equation. Subsequently, the expression of total deflection is obtained and the maximum deflection can be determined. The safety factor is defined according to the Hoff safety criterion to calculate the failure probability of structure. On the basis of the aforementioned analytical model, Monte Carlo method is applied to simulate the random response of buckling behavior, to predict the range of maximum defection and to give the failure probability of structure. The finite element method is also applied to simulate the buckling behavior of plate with initial geometrical imperfection. The interval of deflection calculated by finite element simulation lies in that obtained by Monte Carlo simulation. The analysis of this paper provides a new analytical model for the buckling problem of plate with uncertain initial geometrical imperfection. This method is practical since only the information of manufacturing process error is needed and the calculating process is easy to achieve. 


\section{REFERENCES}

[1] MORGAN EJ, Seide P, WEINGARTEN VI. Elastic stability of thin-walled cylindrical and conical shells under axial compression. Aiaa J 1965;3(3):500-5.

[2] Papadopoulos V, Papadrakakis $M$. The effect of material and thickness variability on the buckling load of shells with random initial imperfections. Comput Method Appl M 2005 2005-04-08;194(12-16):1405-26.

[3] Broggi M, Schuëller GI. Efficient modeling of imperfections for buckling analysis of composite cylindrical shells. Eng Struct 2011;33(5):1796-806.

[4] Gamez-Montero PJ, Salazar E, Castilla R, Freire J, Khamashta M, Codina E. Misalignment effects on the load capacity of a hydraulic cylinder. Int J Mech Sci 2009;51(2):105-13.

[5] Ifayefunmi O, Błachut J. The Effect of Shape, Thickness and Boundary Imperfections on Plastic Buckling of Cones. ASME 2011 30th International Conference on Ocean, Offshore and Arctic Engineering; 2011: American Society of Mechanical Engineers; 2011. p. 23-33.

[6] Zhang J, Ellingwood B. Effects of uncertain material properties on structural stability. Journal of structural engineering 1995;121(4):705-16.

[7] Pocratsky RM, de Boer MP. Self-tensioning Support Post Design to Control Residual Stress in MEMS Fixed-Fixed Beams. MRS Proceedings; 2014: Cambridge Univ Press; 2014. p. f13-1659.

[8] Papadopoulos V, Soimiris G, Papadrakakis M. Buckling analysis of I-section portal frames with stochastic imperfections. Eng Struct 2013;47:54-66.

[9] Park YS, Oh JJ, Kim KS, Park JS. A Study on Inelastic Lateral-Torsional Buckling Strengths with Load Height Effects. Journal of korean society of hazard mitigation 2012;12(1):61-7.

[10]Ellinas CP, Supple WJ, Walker AC. Buckling of Offshore Structures: A State-of-the-art-review of the Buckling of Offshore Structures: Granada, 1984.

[11]Schenk CA, Schuëller GI. Buckling analysis of cylindrical shells with random geometric imperfections. Int J Nonlinear Mech 2003;38(7):1119-32.

[12]Tomás A, Tovar JP. The influence of initial geometric imperfections on the buckling load of single and double curvature concrete shells. Comput Struct 2012;96:34-45.

[13]Ruixiang ZZCH. ROBUST OPTIMIZATION OF ADVANCED GRID COMPOSITE CYLINDRICAL SHELL CONSIDING INITIAL IMPERFECT EFFECT [J]. Acta Mech Solida Sin 2006;1:9.

[14]Kala Z. Stability problems of steel structures in the presence of stochastic and fuzzy uncertainty. Thin Wall Struct 2007;45(10):861-5.

[15]Flügge W. Die stabilität der kreiszylinderschale. Arch Appl Mech 1932;3(5):463-506.

[16]Donnell LH, Wan CC. Effect of imperfections on buckling of thin cylinders and columns under axial compression. J Appl Mech-T Asme 1950;17(1):73-83.

[17]Koiter WT. The stability of elastic equilibrium: DTIC Document; 1970.

[18]Hutchinson JW. Plastic buckling.: DTIC Document; 1973.

[19]Cases CS. Theory of Buckling and Post-Buckling Behavior of Elastic Structures. Adv Appl Mech 1975;14:1.

[20]Qiu Z. Convex models and interval analysis method to predict the effect of uncertain-but-bounded parameters on the buckling of composite structures. Comput Method Appl M 2005;194(18):2175-89.

[21]Qiu Z, Wang X. Comparison of dynamic response of structures with uncertain-but-bounded parameters using non-probabilistic interval analysis method and probabilistic approach. Int $\mathrm{J}$ Solids Struct 2003;40(20):5423-39.

[22]Elishakoff I. Impact buckling of thin bar via Monte Carlo method. Journal of Applied Mechanics 1978;45(3):586-90.

[23] Cardoso JB, de Almeida JR, Dias JM, Coelho PG. Structural reliability analysis using Monte Carlo simulation and neural networks. Adv Eng Softw 2008;39(6):505-13.

[24]Hoff NJ. DYNAMIC STABILITY OF STRUCTURES.: DTIC Document; 1965. 